\title{
Minimizing the Makespan for Scheduling Problems with General Deterioration Effects
}

\author{
Xianyu Yu, ${ }^{1,2,3}$ Yulin Zhang, ${ }^{1}$ and Kai Huang ${ }^{2}$ \\ ${ }^{1}$ School of Economics and Management, Southeast University, Nanjing, Jiangsu 211189, China \\ ${ }^{2}$ DeGroote School of Business, McMaster University, Hamilton, ON, Canada L8S 4M4 \\ ${ }^{3}$ School of Sciences, East China Institute of Technology, Fuzhou, Jiangxi 344000, China \\ Correspondence should be addressed to Yulin Zhang; zhangyl@seu.edu.cn
}

Received 29 August 2013; Accepted 19 November 2013

Academic Editor: Yunqiang Yin

Copyright (C) 2013 Xianyu Yu et al. This is an open access article distributed under the Creative Commons Attribution License, which permits unrestricted use, distribution, and reproduction in any medium, provided the original work is properly cited.

This paper investigates the scheduling problems with general deterioration models. By the deterioration models, the actual processing time functions of jobs depend not only on the scheduled position in the job sequence but also on the total weighted normal processing times of the jobs already processed. In this paper, the objective is to minimize the makespan. For the singlemachine scheduling problems with general deterioration effects, we show that the considered problems are polynomially solvable. For the flow shop scheduling problems with general deterioration effects, we also show that the problems can be optimally solved in polynomial time under the proposed conditions.

\section{Introduction}

In the last decade, there is a growing interest in the literatures to study the scheduling problems where the actual processing time of a job may be subject to change due to various possible changes of its starting time and/or its position in the scheduling sequence. In general, there are two main types of effects on actual processing time of jobs in the scheduling literature: position-based effect and sum-of-processing-timebased effect.

Many studies modeled the actual processing times of jobs as specific exponential functions of the scheduled positions of jobs. Biskup [1] considered a scheduling problem with an exponential model of position-dependent effect where the actual processing time of job $J_{i}$ is

$$
p_{i r}=p_{i} r^{a}
$$

if job $J_{i}$ is scheduled in position $r$ of the job sequence, where $p_{i}$ and $a(a<0)$ are the normal processing time and the learning rate of job $J_{i}$, respectively. The model in Biskup [1] was extended to be

$$
p_{i r}=p_{i} r^{a_{i}}
$$

where the learning rates of the jobs are different; that is, the learning effects are job dependent in Mosheiov and Sidney [2]. Some recent studies (see, e.g., Kuo and Yang [3], Cheng et al. [4], Chang et al. [5], Yang et al. [6], and Browne and Yechiali [7]) have investigated this issue with exponential processing time models under different machine environments. Some other studies proposed different versions of linear processing time models of jobs. Zhao and Tang [8] considered a linear deterioration model in which the actual processing time of job $J_{j}$ is

$$
p_{j}^{A}=p_{j}+a_{j} t
$$

where $p_{j}, a_{j}$, and $t$ are the normal processing time, deterioration rate, and starting time of job $J_{i}$, respectively. Cheng and 
Wang [9] introduced an actual processing time model of $J_{i}$ as follows:

$$
p_{j}=a_{i}-v_{i} \min \left\{n_{i}, n_{0 i}\right\}, \quad i=1,2, \ldots, n,
$$

where $p_{j}$ and $v_{i}$ are the normal processing time of job $J_{i}$ and the learning effect coefficient indicating the relative degree of learning, respectively. The notation $n_{i}$ is a nonnegative integer such that $0 \leq n_{i} \leq n-1$ which indicates the number of jobs processed before job $J_{i}$ in the schedule (i.e., $n_{i}+1$ is the position of job $J_{i}$ in the schedule), and the notation $n_{0 i}$ is a nonnegative integer such that $n_{0 i} \leq n-1$ indicating a threshold value. The scheduling problems with linear actual processing times of jobs were considered in many recent articles, which include Mosheiov [10], Bachman and Janiak [11], Li et al. [12], Cheng et al. [13], and Yin et al. [14].

In the last decade, many researchers have devoted themselves to address different combinations of nonlinear models and linear models for actual processing times of jobs in scheduling studies. Alidaee and Womer [15] proposed a simple processing time model of job $J_{j}$ as follows:

$$
p_{j r}(t)=p_{j}+\alpha t^{a}
$$

where $a>0$ and $\alpha>0$ are both parameters of the nonlinear deterioration effect. Lee et al. [16] considered the bicriterion single-machine scheduling problem with two job processing time models as follows:

$$
\begin{gathered}
p_{j r}(t)=\alpha_{j} t r^{a}, \\
p_{j r}(t)=\left(p_{0}+\alpha_{j} t\right) r^{a},
\end{gathered}
$$

where $\alpha_{j}$ and $t$ are the deterioration rate of job $J_{j}$ and the starting time of job $J_{j}$, respectively.

Several researchers addressed the combined effect of position-based effect and sum-of-processing-time-based effect into various scheduling problems. S.-J. Yang and D.-L. Yang [17] proposed a model in which the actual processing time of job $J_{j}$ scheduled in position $r$ of a sequence is

$$
p_{i r}=p_{i}\left(1+\frac{\sum_{k=1}^{r-1} p_{[k]}}{\sum_{k=1}^{n} p_{k}}\right)^{a_{1}} r^{a_{2}},
$$

where $a_{1}<0$ and $a_{2}<0$ denote the learning rates for sumof-processing-times-based and position-based learning, respectively. In the model, the subscript $[k]$ denotes the job in position $k$ of a sequence, and $p_{[i]}$ denotes the normal processing time of job $i$. Yin et al. [18] extended the model in S.-J. Yang and D.-L. Yang [17] and proposed another learning model in which the actual processing time of job $J_{j}$ scheduled in position $r$ of a sequence is

$$
p_{i r}=p_{i} f\left(\sum_{k=1}^{r-1} p_{[k]}\right) g(r),
$$

where $\sum_{k=1}^{0} p_{[k]}=0, f:[0,+\infty) \rightarrow(0,1]$ is a differentiable nonincreasing function where $f$ is nondecreasing on $[0,+\infty), f(0)=1$, and $g:[0,+\infty) \rightarrow(0,1]$ is a nonincreasing function with $g(1)=1$. Many recent studies (see, e.g., Wang [19], Toksarı and Güner [20], Yang and Kuo [21], Cheng et al. [22, 23], Cheng et al. [24], Kuo [25], Yang [26], and Lee and $\mathrm{Wu}$ [27]) have been conducted to address the combined processing time models in scheduling problems.

In a recent paper, J.-B. Wang and J.-J. Wang [28] proposed a new general learning effect model for both position-based effect and sum-of-processing-time-based effect, which can be described as

$$
p_{i r}=p_{i} f\left(\sum_{l=1}^{r-1} \beta_{l} p_{[l]}\right) g(r)
$$

where $\sum_{l=1}^{0} \beta_{l} p_{[l]}:=0, f:[0,+\infty) \rightarrow(0,1]$ is a differentiable nonincreasing function where $f^{\prime}$ is nondecreasing on $[0,+\infty), g:[1,+\infty) \rightarrow(0,1]$ is a nonincreasing function with $g(1)=1$, and $\beta_{l}$ is the weight of position $r$. They showed that even with the introduction of a general learning effect to job processing times, some single-machine scheduling problems can be optimally solved in polynomial time. They also proved that some special cases of the flow shop scheduling problems are polynomially solvable under the proposed model.

In this paper, we explore to consider scheduling problems with considerations of two general deterioration effects whose actual processing times functions of jobs are modified from the models in Lee and Wu [27] and J.-B. Wang and J.-J. Wang [28] with respect to deterioration effects. The motivation for our study stems from the hardware production line that cuts a class of products to proper sizes and shapes. The actual processing time of a product depends on the quality of the cutting tool in the manufacturing process. Due to tool blunting, the actual processing time of a product increases with respect to the sum of the processing time of products already processed by the cutting tool and the number of products already processed. The objective of our study is to find the optimal job sequence to minimize the makespan of all the jobs.

The remaining part of this paper is organized as follows. In Section 2, we consider two single-machine scheduling problems. In Section 3, we investigate the flow shop scheduling problems under proper given conditions. We conclude this paper and describe the possible extensions in the last section.

\section{Single-Machine Scheduling}

The formulation of the considered single-machine scheduling problems can be described as follows. At time zero, there are $n$ independent jobs ready to be processed on a singlemachine. Preemption is not allowed and a machine is only able to process one job at a time. Job $j$ has a normal processing time $p_{j}(j=1,2, \ldots, n)$.

In this paper, we propose two general deterioration models with respect to both position-based effect and sumof-processing-time-based effect. By the first model, if job $j$ 
is scheduled in the $r$ position of the sequence, the actual processing time of job $j$ is

$$
p_{j r}=p_{j}\left[f\left(\sum_{l=1}^{r-1} \beta_{l} p_{[l]}\right)+g(r)\right],
$$

where $\sum_{l=1}^{0} \beta_{l} p_{[i, l]}:=0, f:[0,+\infty) \rightarrow[1,+\infty)$ is a differentiable nondecreasing function and $f(0)=1, g:[1,+\infty) \rightarrow$ $[1,+\infty)$ is a nondecreasing function with $g(1)=1, \beta_{l}$ is the weight of position $l, p_{[l]}$ denotes the normal processing time of job $i$, and the subscript $[l]$ denotes the job in position $l$ of a sequence. In some actual processes, different jobs always have different complexities which lead to different experience to the worker in production line; thus, we assume that $0 \leq \beta_{1} \leq$ $\beta_{2} \leq \cdots \beta_{n}$.

By the second model, if job $j$ is scheduled in the $r$ position, its actual processing time is

$$
p_{j r}=p_{j} f\left(\sum_{l=1}^{r-1} \beta_{l} p_{[l]}\right) g(r),
$$

where all the notations have the same means as those of the first model. Note that the function used in the model (11) is the same as the function used by J.-B. Wang and J.-J. Wang [28]. The difference between this model and the model in J.-B. Wang and J.-J. Wang [28] is that the former aims to study the deterioration effect while the latter (J.-B. Wang and J.-J. Wang [28]) considers learning effect.

By using the three-field notation scheme for the scheduling problem introduced by Graham et al. [29], the singlemachine scheduling problem with respect to the first model can be denoted as $1 \mid p_{j r}=p_{j}\left[f\left(\sum_{l=1}^{r-1} \beta_{l} p_{[l]}\right)+g(r)\right]$, DE | $C_{\text {max }}$, where DE denotes the deterioration effect. Similarly, the single-machine scheduling problem with respect to the second model can be denoted as $1 \mid p_{j r}=p_{j} f\left(\sum_{l=1}^{r-1} \beta_{l} p_{[l]}\right) g(r)$, $\mathrm{DE} \mid \mathrm{C}_{\max }$.

In the following, we first state two lemmas that will be used in the proofs of the following theorems in this section.

Lemma 1. If $f:[0,+\infty) \rightarrow[1,+\infty)$ is nondecreasing and its derived function $f^{\prime}$ is nonincreasing on $[0,+\infty), \lambda \geq 1, \delta_{1} \geq 1$, and $x \geq 0$, then $(1-\lambda)\left(f(A) \delta_{2}+1\right)+\lambda\left(f(A+x) \delta_{2}+\delta_{1}\right)-$ $\left(f(a+\lambda x) \delta_{2}+\delta_{1}\right) \geq 0$.

Proof. We let $h(x)=(1-\lambda)\left(f(A) \delta_{2}+1\right)+\lambda\left(f(A+x) \delta_{2}+\right.$ $\left.\delta_{1}\right)-\left(f(A+\lambda x) \delta_{2}+\delta_{1}\right)$. The first derivation of $h(x)$ with respect to $x$ can be obtained that $h^{\prime}(x)=\lambda \delta_{2}\left[f^{\prime}(A+x)-\right.$ $\left.f^{\prime}(A+\lambda x)\right] \geq 0$ under the condition that $f:[0,+\infty) \rightarrow$ $[1,+\infty)$ is a differentiable nondecreasing function with $f^{\prime}$ being nonincreasing on $[0,+\infty), \lambda \geq 1$, and $\delta_{1} \geq 1$. Then, it is obvious that $h(x)$ is an increasing function on $x$. Therefore, we have $h(x) \geq h(0)=(1-\lambda)\left(1-\delta_{1}\right) \geq 0$ for $\lambda \geq 1, \delta_{1} \geq 1$.

With a similar differentiation method, it is easy to obtain the following lemma.

Lemma 2. If $f:[0,+\infty) \rightarrow[1,+\infty)$ is nondecreasing and its derived function $f^{\prime}$ is nonincreasing on $[0,+\infty), \lambda \geq 1, \delta \geq 1$, and $x \geq 0$, then $(1-\lambda) f(A)+\lambda \delta f(A+x)-\delta f(A+\lambda x) \geq 0$.
Theorem 3. The problem $1 \mid p_{j r}=p_{j}\left(f\left(\sum_{l=1}^{r-1} \beta_{l} p_{[l]}\right)+g(r)\right)$, $D E \mid C_{\max }$ can be optimally solved by the Longest Processing Time first rule of $p_{j}$ (the LPT rule) if $f^{\prime}$ is nonincreasing on $[0,+\infty)$.

Proof. To prove this theorem, it is sufficient to prove that a schedule can be enhanced by scheduling the job with longer processing time into the earlier position of the schedule under the given condition in the theorem. Let the schedule $\sigma$ be $\left[\sigma_{1}, J_{j}, J_{k}, \sigma_{i 2}, \sigma_{2}\right]$ and let the schedule $\sigma^{\prime}$ be $\left[\sigma_{1}, J_{k}, J_{j}, \sigma_{2}\right]$, where $\sigma_{1}$ and $\sigma_{2}$ are partial job sequences, and the difference between $\sigma$ and $\sigma^{\prime}$ is a pairwise interchange of two adjacent jobs $J_{j}$ and $J_{k}$ in the schedule. Concretely, $J_{j}$ and $J_{k}$ are assumed to be assigned in the $r$ th and $r+1$ th position, respectively, in the schedule $\sigma$. In $\sigma^{\prime}, J_{j}$ and $J_{k}$ are assigned in the $r+$ 1 th and $r$ th position, respectively. Without loss of generality, the normal processing times of $J_{j}$ and $J_{k}$ are assumed to satisfy $p_{j} \leq p_{k}$. To show that $\sigma^{\prime}$ dominates $\sigma$, it suffices to show that $C_{k}(\sigma) \geq C_{j}\left(\sigma^{\prime}\right)$ and $C_{u}(\sigma) \geq C_{u}\left(\sigma^{\prime}\right)$ for any job $J_{u}$ in $\sigma_{2}$. Let the completion time of the last job in partial schedule $\sigma_{1}$ be $C_{\sigma_{1}}$. In the schedule $\sigma$, the completion times of $J_{j}$ and $J_{k}$ are

$$
\begin{array}{r}
C_{j}(\sigma)=C_{\sigma_{1}}+p_{j}\left[f\left(\sum_{l=1}^{r-1} \beta_{l} p_{[l]}\right)+g(r)\right], \\
C_{k}(\sigma)=C_{\sigma_{1}}+p_{j}\left[f\left(\sum_{l=1}^{r-1} \beta_{l} p_{[l]}\right)+g(r)\right] \\
+p_{k}\left[f\left(\sum_{l=1}^{r-1} \beta_{l} p_{[l]}+\beta_{r} p_{j}\right)+g(r+1)\right],
\end{array}
$$

respectively. In the schedule $\sigma^{\prime}$, the completion times of $J_{k}$ and $J_{j}$ are

$$
\begin{array}{r}
C_{k}\left(\sigma^{\prime}\right)=C_{\sigma_{1}}+p_{k}\left[f\left(\sum_{l=1}^{r-1} \beta_{l} p_{[l]}\right)+g(r)\right] \\
C_{j}\left(\sigma^{\prime}\right)=C_{\sigma_{1}}+p_{k}\left[f\left(\sum_{l=1}^{r-1} \beta_{l} p_{[l]}\right)+g(r)\right] \\
+p_{j}\left[f\left(\sum_{l=1}^{r-1} \beta_{l} p_{[l]}+\beta_{r} p_{k}\right)+g(r+1)\right],
\end{array}
$$

respectively. Combining (13) and (15), we have

$$
\begin{aligned}
C_{k}(\sigma) & -C_{j}\left(\sigma^{\prime}\right) \\
= & \left(p_{j}-p_{k}\right)\left[f\left(\sum_{l=1}^{r-1} \beta_{l} p_{[l]}\right)+g(r)\right] \\
+ & p_{k}\left[f\left(\sum_{l=1}^{r-1} \beta_{l} p_{[l]}+\beta_{r} p_{j}\right)+g(r+1)\right] \\
& -p_{j}\left[f\left(\sum_{l=1}^{r-1} \beta_{l} p_{[l]}+\beta_{r} p_{k}\right)+g(r+1)\right]
\end{aligned}
$$




$$
\begin{gathered}
\times \frac{p_{k}}{p_{j}}\left[f\left(\sum_{l=1}^{r-1} \beta_{l} p_{[l]}\right) \frac{1}{g(r)}+1\right] \\
=p_{j} g(r)\left\{\left(1-\frac{p_{k}}{p_{j}}\right)\left[f\left(\sum_{l=1}^{r-1} \beta_{l} p_{[l]}\right) \frac{1}{g(r)}+1\right]\right. \\
+\frac{p_{k}}{p_{j}}\left[f\left(\sum_{l=1}^{r-1} \beta_{l} p_{[l]}+\beta_{r} p_{j}\right)\right. \\
-\left[f\left(\sum_{l=1}^{r-1} \beta_{l} p_{[l]}+\beta_{r} p_{j} \frac{p_{k}}{p_{j}}\right)\right. \\
\left.\times \frac{1}{g(r)}+\frac{g(r+1)}{g(r)}\right]
\end{gathered}
$$

Let $A=\sum_{l=1}^{r-1} \beta_{l} p_{[l]}, \lambda=p_{k} / p_{j}, \delta_{1}=(g(r+1) / g(r)), \delta_{2}=$ $(1 / g(r))$, and $x=\beta_{r} p_{j}$. Based on the formulation of the considered problem, it is easy to obtain that $A \geq 0, \lambda \geq 1, \delta_{1} \geq$ $1,0<\delta_{2} \leq 1$, and $x \geq 0$. Based on Lemma 1 and (16), we have

$$
\begin{aligned}
C_{k}(\sigma)-C_{j}\left(\sigma^{\prime}\right) & \\
=p_{j} g(r)[ & (1-\lambda)\left(f(A) \delta_{2}+1\right) \\
+ & \lambda\left(f(A+x) \delta_{2}+\delta_{1}\right) \\
& \left.-\left(f(a+\lambda x) \delta_{2}+\delta_{1}\right)\right] \geq 0 .
\end{aligned}
$$

Assuming that $J_{s}$ is the job scheduled in the $r+2$ position of the job sequence, then the completion times of $J_{s}$ in $\sigma$ and $\sigma^{\prime}$ are

$$
\begin{aligned}
C_{s}(\sigma)=C_{k}(\sigma) & \\
+ & p_{s}\left[f\left(\sum_{l=1}^{r-1} \beta_{l} p_{[i, l]}+\beta_{r} p_{j}+\beta_{r+1} p_{k}\right)\right. \\
& +g(r+2)], \\
C_{s}\left(\sigma^{\prime}\right)=C_{j}\left(\sigma^{\prime}\right) & \\
+ & p_{s}\left[f\left(\sum_{l=1}^{r-1} \beta_{l} p_{[i, l]}+\beta_{r} p_{k}+\beta_{r+1} p_{j}\right)\right. \\
& +g(r+2)],
\end{aligned}
$$

respectively. Since $p_{j} \leq p_{k}$ and $\beta_{r} \leq \beta_{r+1}$, it can be obtained that

$$
\begin{gathered}
\left(\beta_{r} p_{j}+\beta_{r+1} p_{k}\right)-\left(\beta_{r} p_{k}+\beta_{r+1} p_{j}\right) \\
=\left(\beta_{r}-\beta_{r+1}\right)\left(p_{j}-p_{k}\right) \geq 0
\end{gathered}
$$

Since $f$ is a nondecreasing function, $(20)$ is equivalent to

$$
\begin{aligned}
& f\left(\sum_{l=1}^{r-1} \beta_{l} p_{[i, l]}+\beta_{r} p_{j}+\beta_{r+1} p_{k}\right) \\
& \quad \geq f\left(\sum_{l=1}^{r-1} \beta_{l} p_{[i, l]}+\beta_{r} p_{k}+\beta_{r+1} p_{j}\right) .
\end{aligned}
$$

Let $C_{s}(\sigma)$ and $C_{s}\left(\sigma^{\prime}\right)$ denote the completion time of job $J_{s}$ in the schedule $\sigma$ and $\sigma^{\prime}$, respectively. From (17)-(19) and (21), we have $C_{s}(\sigma) \geq C_{s}\left(\sigma^{\prime}\right)$. Thus, we show that $J_{s}$ starts earlier in $\sigma^{\prime}$ than in $\sigma$ and completes earlier in $\sigma^{\prime}$ than in $\sigma$. With a similar proof method, it can be shown that $C_{u}(\sigma) \geq C_{u}\left(\sigma^{\prime}\right)$ for any job $J_{u}$ in $\sigma_{2}$. By repeating the above argument, it is obvious to lead to the conclusion that a schedule can be enhanced by scheduling the job with longer processing time into the earlier position of the job sequence.

Based on Lemma 2, the following theorem can be obtained by using a similar proof method to that of Theorem 3.

Theorem 4. The problem $1 \mid p_{j r}=p_{j}\left(f\left(\sum_{l=1}^{r-1} \beta_{l} p_{[l]}\right) g(r)\right)$, $D E \mid C_{\max }$ can be optimally solved by the Longest Processing Time first rule (the LPT rule) if $f^{\prime}$ is nonincreasing on $[0,+\infty)$.

If $g(r)$ is unchanged with respect to $r$, then two actual processing time models can be both simplified as $p_{j r}=p_{j} f$ $\left(\sum_{l=1}^{r-1} \beta_{l} p_{[l]}\right)$. Similarly, if $f\left(\sum_{l=1}^{r-1} \beta_{l} p_{[l]}\right)$ is unchanged with respect to $\sum_{l=1}^{r-1} \beta_{l} p_{[l]}$, then two actual processing time models can be both simplified as $p_{j r}=p_{j} g(r)$. It is obvious that the single-machine problem with the above special models can be also optimally solved by the LPT rule.

\section{Flow Shop Scheduling}

The formulation of the flow shop scheduling problem can be described as follows. There are $n$ independent jobs $J_{1}, J_{2}, \ldots, J_{n}$ ready to be processed on $m$ machines $M_{1}, M_{2}, \ldots, M_{m}$ at time zero. Job $J_{j}$ consists of $m$ operations $\mathrm{O}_{1 j}, \mathrm{O}_{2 j}, \ldots, \mathrm{O}_{m j}$. Preemption is not allowed and a machine is only able to process one operation at a time. All machines should process the operations of the jobs in the same order. Operation $O_{i+1, j}$ may start only after operation $O_{i j}$ has been completed. Operation $O_{i j}$ has a normal processing time $p_{i j}(i=1,2, \ldots, m ; j=1,2, \ldots, n)$, and the actual processing time of operation $O_{i j}$ is $p_{i j r}$ if it is scheduled in position $r$ $(r=1,2, \ldots, n)$ in a sequence. In this paper, we propose two general deterioration models. By the first model, if operation $O_{i j}$ is scheduled in the $r$ position of the sequence, the actual processing time of operation $O_{i j}$ is

$$
p_{i j r}=p_{i j}\left[f\left(\sum_{l=1}^{r-1} \beta_{l} p_{[i l]}\right)+g(r)\right],
$$

where $\sum_{l=1}^{0} \beta_{l} p_{[i, 1]}:=0, f:[0,+\infty) \rightarrow[1,+\infty)$ is a differentiable nondecreasing function, and $f(0)=1, g$ : $[1,+\infty) \rightarrow[1,+\infty)$ is a nonincreasing function with 


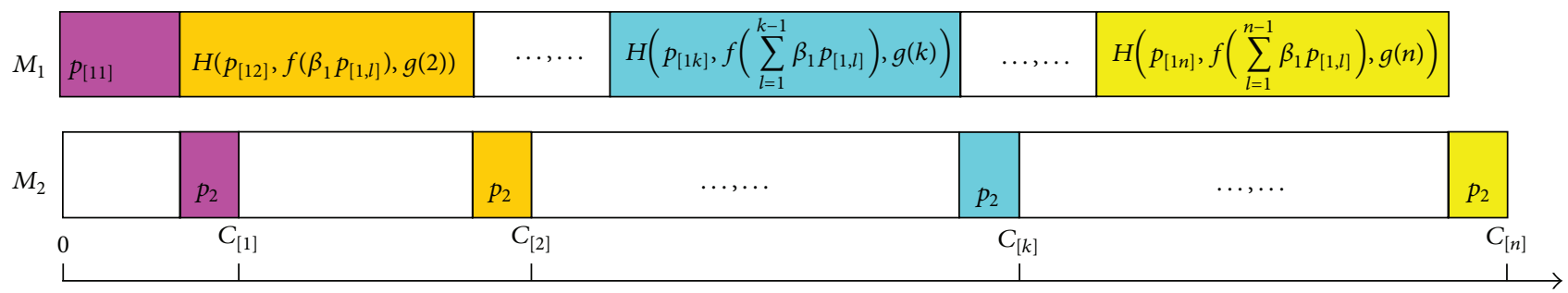

FIGURE 1: The illustration of the problem $F\left|p_{i j r}=H\left(p_{i j}, f\left(\sum_{l=1}^{r-1} \beta_{l} p_{[i l]}\right), g(r)\right), p_{2 j}=p_{2}, \mathrm{DE}\right| C_{\max }$.

$g(1)=1$, and $\beta_{l}$ is the weight of position $l ; p_{[i l]}$ denotes the normal processing time of the operation scheduled in the $r$ th position on machine $M_{i}$, where the subscript $[i, l]$ denotes the job in position $l$ of the sequence on $M_{i}$. In many real life scenarios, positions are characterized by a different grade of complexity; thus, each position can provide different complexity to the manufacturing process; hence, we assume that $0 \leq \beta_{1} \leq \beta_{2} \cdots \beta_{n}$.

By the second model, if job $j$ is scheduled on the $r$ position of machine $i$, its actual processing time is

$$
p_{i j r}=p_{i j} f\left(\sum_{l=1}^{r-1} \beta_{l} p_{[i l]}\right) g(r),
$$

where all the notations have the same means as those of the first model.

In the following, we first introduce a new general deterioration model. The actual processing time of job $j$ on the $r$ position of machine $i$ is

$$
p_{i j r}=H\left[p_{i j}, f\left(\sum_{l=1}^{r-1} \beta_{l} p_{[i l]}\right), g(r)\right] \text {, }
$$

where $p_{i j 1}=H\left(p_{i j}, f(0), g(1)\right)=p_{i j}$ and $H$ is nondecreasing in $p_{i j}, f\left(\sum_{l=1}^{r-1} \beta_{l} p_{[i l]}\right)$, and $g(r)$. Obviously, model (22) and model (23) are both special cases of new model (24).

Lemma 5. The problem $F \mid p_{i j r}=H\left[p_{i j}, f\left(\sum_{l=1}^{r-1} \beta_{l} p_{[i l]}\right)\right.$, $g(r)], p_{2 j}=p_{2}, D E \mid C_{\max }$ has the same optimal schedule as the problem $1\left|p_{1 j r}=H\left[p_{1 j}, f\left(\sum_{l=1}^{r-1} \beta_{l} p_{[1 l]}\right), g(r)\right], D E\right|$ $C_{\max }$ if $p_{1 j} \geq p_{2}$.

Proof. Let $C_{[j]}$ denote the completion time of the job scheduled in $j$ th position of the job sequence. For $j=1$, we have $C_{[1]}=p_{[11]}+p_{2}$.

Because $p_{2 j}=p_{2}$ and $p_{1 j} \geq p_{2}$, it can be obtained that $p_{1 j r}=H\left[p_{1 j}, f\left(\sum_{l=1}^{r-1} \beta_{l} p_{[1]]}\right), g(r)\right] \geq p_{2}$ for $j=1,2, \ldots, n$ and $r=1,2, \ldots, n$. The schedule is illustrated in Figure 1.
Then, we have the completion times for jobs $j=2,3, \ldots, n$ in the following:

$$
\begin{aligned}
& C_{[2]} \\
& =\max \left\{C_{[1]}, p_{[11]}+H\left[p_{[12]}, f\left(\beta_{1} p_{[1, l]}\right), g(2)\right]\right\} p_{[11]} \\
& +p_{2} \\
& =p_{[11]}+H\left(p_{[12]}, f\left[\beta_{1} p_{[1, l]}\right), g(2)\right]+p_{2}, \\
& \vdots \\
& C_{[k+1]} \\
& =\max \left\{C_{[k]}, \sum_{r=1}^{k} H\left[p_{[1 r]}, f\left(\sum_{l=1}^{r-1} \beta_{l} p_{[1, l]}\right), g(r)\right]\right\} \\
& \times p_{[11]}+p_{2} \\
& =\sum_{r=1}^{k} H\left[p_{[1 r]}, f\left(\sum_{l=1}^{r-1} \beta_{l} p_{[1, l]}\right), g(r)\right]+p_{2}, \\
& \vdots \\
& C_{\max } \\
& =C_{[n]} \\
& =\max \left\{C_{[k]}, \sum_{r=1}^{k} H\left[p_{[1 r]}, f\left(\sum_{l=1}^{r-1} \beta_{l} p_{[i, l]}\right), g(r)\right]\right\} \\
& \times p_{[11]}+p_{2} \\
& =\sum_{r=1}^{n} H\left[p_{[1 r]}, f\left(\sum_{l=1}^{r-1} \beta_{l} p_{[1, l]}\right), g(r)\right]+p_{2} \text {. }
\end{aligned}
$$

Since $p_{2}$ is constant, an optimal schedule of minimizing the makespan for the problem $F 2 \mid p_{1 j r}=H\left[p_{1 j}, f\left(\sum_{l=1}^{r-1} \beta_{l} p_{[1 l]}\right.\right.$, $g(r)$ ], DE | $C_{\max }$ must be the optimal schedule of minimizing for the problem $F 2 \mid p_{i j r}=H\left[p_{i j}, f\left(\sum_{l=1}^{r-1} \beta_{l} p_{[1 l]}\right), g(r)\right], p_{2 j}=$ $p_{2}, \mathrm{DE} \mid C_{\max }$.

With a similar proof method of Lemma 5, we can obtain the following theorem. 
Theorem 6. The problem $\mathrm{Fm} \mid p_{i j r}=H\left[p_{i j}, f\left(\sum_{l=1}^{r-1} \beta_{l} p_{[i l]}\right)\right.$, $g(r)], p_{2 j r}=p_{3 j r}=\cdots=p_{m j r}=p, D E \mid C_{\max }$ has the same optimal schedule as the problem $1 \mid p_{1 j r}=H\left(p_{1 j}\right.$, $f\left(\sum_{l=1}^{r-1} \beta_{l} p_{[1]]}, g(r)\right), D E \mid C_{\max }$ if $p_{1 j} \geq p$. ollary.

Combining Theorems 3 and 6 , we have the following cor-

Corollary 7. The problem $\mathrm{Fm} \mid p_{i j r}=p_{i j}\left[f\left(\sum_{l=1}^{r-1} \beta_{l} p_{[i]}\right)+\right.$ $g(r)], p_{2 j r}=p_{3 j r}=\cdots=p_{m j r}=p, D E \mid C_{\max }$ can be optimally solved by the Longest Processing Time first rule of $p_{j}$ (the LPT rule) if $f^{\prime}$ is nonincreasing on $[0,+\infty)$ and $p_{1 j} \geq p$.

Similarly, based on Theorems 4 and 6 , it is easy to get the following result.

Corollary 8. The problem $F m \mid p_{i j r}=p_{i j} f\left(\sum_{l=1}^{r-1} \beta_{l} p_{[i l]}\right) g(r)$, $p_{2 j r}=p_{3 j r}=\cdots=p_{m j r}=p, D E \mid C_{\max }$ can be optimally solved by the Longest Processing Time first rule of $p_{j}$ (the LPT rule) if $f^{\prime}$ is nonincreasing on $[0,+\infty)$ and $p_{1 j} \geq p$.

Theorem 9. If a schedule simultaneously minimizes $H\left(p_{[2 n]}\right.$, $\left.f\left(\sum_{l=1}^{n-1} \beta_{l} p_{[2, l]}\right), g(n)\right)$ and the makespan of the problem 1 । $p_{1 j r}=H\left(p_{1 j}, f\left(\sum_{l=1}^{r-1} \beta_{l} p_{[1 l]}, g(r)\right), D E \mid C_{\max }\right.$, it must be the optimal schedule of the problem F2 | $p_{i j r}=H\left(p_{i j}\right.$, $f\left(\sum_{l=1}^{r-1} \beta_{l} p_{[i l]}, g(r)\right), \min \left(p_{1}.\right) \geq \max \left(p_{2}.\right), D E \mid C_{\max }$.

Proof. By using the same notation as that in the proof of Theorem 6, we let $C_{[j]}$ denote the completion time of the job scheduled in $j$ th position of the job sequence. For $j=1$, we have $C_{[1]}=p_{[11]}+p_{2}$.

Because $\min \left(p_{1}.\right) \geq \max \left(p_{2}.\right)$, it can be obtained that

$$
\begin{aligned}
& H\left(p_{1 j}, f\left(\sum_{l=1}^{r-1} \beta_{l} p_{[1 l]}\right), g(r)\right) \\
& \quad \geq H\left(p_{2 j}, f\left(\sum_{l=1}^{r-1} \beta_{l} p_{[2 l]}\right), g(r)\right)
\end{aligned}
$$

for $j=1,2, \ldots, n$ and $r=1,2, \ldots, n$. Then, we have the completion times for jobs $j=2,3, \ldots, n$ in the following:

$$
\begin{aligned}
C_{[2]}= & \max \left\{C_{[1]}, p_{[11]}+H\left(p_{[12]}, f\left(\beta_{1} p_{[1, l]}\right), g(2)\right)\right\} \\
& +H\left(p_{[22]}, f\left(\beta_{1} p_{[2,1]}\right), g(2)\right) \\
= & p_{[11]}+H\left(p_{[12]}, f\left(\beta_{1} p_{[1, l]}\right), g(2)\right) \\
& +H\left(p_{[22]}, f\left(\beta_{1} p_{[2,1]}\right), g(2)\right),
\end{aligned}
$$

$$
\begin{aligned}
C_{[k+1]}= & \max \left\{C_{[k]}, \sum_{r=1}^{k+1} H\left(p_{[1 r]}, f\left(\sum_{l=1}^{r-1} \beta_{l} p_{[1, l]}\right), g(r)\right)\right\} \\
& +H\left(p_{[2, k+1]}, f\left(\sum_{l=1}^{k} \beta_{l} p_{[2, l]}\right), g(k+1)\right) \\
= & \sum_{r=1}^{k+1} H\left(p_{[1 r]}, f\left(\sum_{l=1}^{r-1} \beta_{l} p_{[1, l]}\right), g(r)\right) \\
& +H\left(p_{[2, k+1]}, f\left(\sum_{l=1}^{k} \beta_{l} p_{[2, l]}\right), g(k+1)\right), \\
& \vdots \\
C_{\max }= & C_{[n]} \\
= & \max \left\{C_{[n-1]},\right. \\
& +H\left(p_{[2 n]}, f\left(\sum_{l=1}^{n-1} \beta_{l} p_{[2, l]}\right), g(n)\right) . \\
= & \left.\sum_{r=1}^{n} H\left(p_{[2 n]}, f\left(\sum_{l=1}^{n-1} \beta_{l} p_{[2, l]}\right), g\left(\sum_{l=1}^{r-1} \beta_{l} p_{[1, l]}\right), g\left(\sum_{l=1}^{r-1} \beta_{l} p_{[1, l]}\right), g(r)\right)\right\} \\
& +g(r)) \\
& \\
& \\
&
\end{aligned}
$$

Since $\sum_{r=1}^{n} H\left(p_{[1 r]}, f\left(\sum_{l=1}^{r-1} \beta_{l} p_{[1, l]}\right), g(r)\right)$ can be explained as the makespan of the problem $1 \mid p_{1 j r}=$ $H\left(p_{1 j}, f\left(\sum_{l=1}^{r-1} \beta_{l} p_{[1]]}, g(r)\right)\right.$, DE | $C_{\text {max }}$, the schedule minimizes $H\left(p_{[2 n]}, f\left(\sum_{l=1}^{n-1} \beta_{l} p_{[2, l]}\right), g(n)\right)$ simultaneously and the makespan of the problem $F 2 \mid p_{1 j r}=H\left(p_{1 j}, f\left(\sum_{l=1}^{r-1} \beta_{l} p_{[1]}\right.\right.$, $g(r))$, DE $\mid C_{\max }$ is the optimal schedule of the problem $F 2 \mid p_{i j r}=H\left(p_{i j}, f\left(\sum_{l=1}^{r-1} \beta_{l} p_{[i l]}, g(r)\right), \min \left(p_{1}\right) \geq \max \left(p_{2}.\right)\right.$, $D E \mid C_{\max }$. ollary.

Combining Theorems 3 and 9, we have the following cor-

Corollary 10. The problem F2 $\mid p_{i j r}=p_{i j}\left[f\left(\sum_{l=1}^{r-1} \beta_{l} p_{[i l]}\right)+\right.$ $g(r)], \min \left(p_{1}.\right) \geq \max \left(p_{2}.\right), D E \mid C_{\max }$ can be optimally solved by the Longest Processing Time first rule of $p_{j}$ (the LPT rule) if $f^{\prime}$ is nonincreasing on $[0,+\infty)$.

Similarly, combining Theorems 4 and 9, we can obtain the following result.

Corollary 11. The problem $F 2 \mid p_{i j r}=p_{i j} f\left(\sum_{l=1}^{r-1} \beta_{l} p_{[i l]}\right) g(r)$, $\min \left(p_{1}\right) \geq \max \left(p_{2}\right), D E \mid C_{\max }$ can be optimally solved by 
the Longest Processing Time first rule of $p_{j}$ (the LPT rule) if $f^{\prime}$ is nonincreasing on $[0,+\infty)$.

\section{Conclusions}

This paper considered three scheduling problems with general deterioration models, where the actual processing time function of a job depends not only on the total weighted normal processing times of jobs already processed but also on the scheduled position in the job sequence. Firstly, for two single-machine scheduling problems with general deterioration models associated with both the total weighted normal processing times of the jobs and the scheduled position in the job sequence, we proved that two singlemachine scheduling problems are both polynomially solvable under the condition that the derived actual processing time functions are nonincreasing, respectively. Secondly, based on the results of the single-machine problem, we also showed that four flow shop scheduling problems can be optimally solved in polynomial time under proposed conditions.

Future works may focus on developing randomized algorithms for the scheduling problem without pregiven conditions. The variability of tool changes or maintenance activities is also worth considering in scheduling problems with general deterioration effects.

\section{Acknowledgments}

The authors wish to thank the editors and the anonymous referees for their helpful comments on the earlier versions of this paper. This work is supported by the National Nature Science Foundation of China (71171046), the National Science and Engineering Research Council of Canada (Discovery Grant CISE-539050), the College Graduate Research and Innovation Foundation of Jiangsu Province (CXLX 0162), and the Scientific Research Foundation of Graduate School of Southeast University (YBJJ1239).

\section{References}

[1] D. Biskup, "Single-machine scheduling with learning considerations," European Journal of Operational Research, vol. 115, no. 1, pp. 173-178, 1999.

[2] G. Mosheiov and J. B. Sidney, "Scheduling with general jobdependent learning curves," European Journal of Operational Research, vol. 147, no. 3, pp. 665-670, 2003.

[3] W.-H. Kuo and D.-L. Yang, "Minimizing the makespan in a single-machine scheduling problem with the cyclic process of an aging effect," Journal of the Operational Research Society, vol. 59, no. 3, pp. 416-420, 2008.

[4] T. C. E. Cheng, C.-C. Wu, J.-C. Chen, W.-H. Wu, and S.-R. Cheng, "Two-machine flowshop scheduling with a truncated learning function to minimize the makespan," International Journal of Production Economics, vol. 141, no. 1, pp. 79-86, 2013.

[5] P.-C. Chang, S.-H. Chen, and V. Mani, "A note on duedate assignment and single machine scheduling with a learning/aging effect," International Journal of Production Economics, vol. 117, no. 1, pp. 142-149, 2009.
[6] S.-J. Yang, D.-L. Yang, and T. C. E. Cheng, "Single-machine due-window assignment and scheduling with job-dependent aging effects and deteriorating maintenance," Computers and Operations Research, vol. 37, no. 8, pp. 1510-1514, 2010.

[7] S. Browne and U. Yechiali, "Scheduling deteriorating jobs on a single processor," Operations Research, vol. 38, no. 3, pp. 495498, 1990.

[8] C.-L. Zhao and H.-Y. Tang, "Single machine scheduling with general job-dependent aging effect and maintenance activities to minimize makespan," Applied Mathematical Modelling, vol. 34 , no. 3, pp. 837-841, 2010.

[9] T. C. E. Cheng and G. Wang, "Single machine scheduling with learning effect considerations," Annals of Operations Research, vol. 98, no. 1-4, pp. 273-290, 2000.

[10] G. Mosheiov, "Scheduling jobs under simple linear deterioration," Computers and Operations Research, vol. 21, no. 6, pp. 653659, 1994.

[11] A. Bachman and A. Janiak, "Scheduling jobs with positiondependentprocessing times," Journal of the Operational Research Society, vol. 55, no. 3, pp. 257-264, 2004.

[12] S. Li, C. T. Ng, and J. Yuan, "Scheduling deteriorating jobs with CON/SLK due date assignment on a single machine," International Journal of Production Economics, vol. 131, no. 2, pp. 747-751, 2011.

[13] T. C. E. Cheng, S.-J. Yang, and D.-L. Yang, "Common duewindow assignment and scheduling of linear time-dependent deteriorating jobs and a deteriorating maintenance activity," International Journal of Production Economics, vol. 135, no. 1, pp. 154-161, 2012.

[14] Y. Q. Yin, T. C. E. Cheng, J. Y. Xu, S.-R. Cheng, and C.C. Wu, "Single-machine scheduling with past-sequence-dependent delivery times and a linear deterioration," Journal of Industrial and Management Optimization, vol. 9, no. 2, pp. 323339, 2013.

[15] B. Alidaee and N. K. Womer, "Scheduling with time dependent processing times: review and extensions," Journal of the Operational Research Society, vol. 50, no. 7, pp. 711-729, 1999.

[16] W.-C. Lee, C.-C. Wu, and H.-J. Sung, "A bi-criterion singlemachine scheduling problem with learning considerations," Acta Informatica, vol. 40, no. 4, pp. 303-315, 2004.

[17] S.-J. Yang and D.-L. Yang, "Single-machine scheduling problems with aging/deteriorating effect under an optional maintenance activity consideration," Information Systems and Operational Research, vol. 48, no. 3, pp. 171-179, 2010.

[18] Y. Yin, D. Xu, K. Sun, and H. Li, "Some scheduling problems with general position-dependent and time-dependent learning effects," Information Sciences, vol. 179, no. 14, pp. 2416-2425, 2009.

[19] J.-B. Wang, "A note on scheduling problems with learning effect and deteriorating jobs," International Journal of Systems Science, vol. 37, no. 12, pp. 827-833, 2006.

[20] M. D. Toksarı and E. Güner, "Minimizing the earliness/tardiness costs on parallel machine with learning effects and deteriorating jobs: a mixed nonlinear integer programming approach," International Journal of Advanced Manufacturing Technology, vol. 38, no. 7-8, pp. 801-808, 2008.

[21] D.-L. Yang and W.-H. Kuo, "Single-machine scheduling with both deterioration and learning effects," Annals of Operations Research, vol. 172, no. 1, pp. 315-327, 2009. 
[22] T. C. E. Cheng, C.-C. Wu, and W.-C. Lee, "Some scheduling problems with sum-of-processing-times-based and jobposition-based learning effects," Information Sciences, vol. 178, no. 11, pp. 2476-2487, 2008.

[23] T. C. E. Cheng, W.-H. Kuo, and D.-L. Yang, "Scheduling with a position-weighted learning effect based on sum-of-logarithmprocessing-times and job position," Information Sciences, vol. 221, pp. 490-500, 2013.

[24] T. C. E. Cheng, W.-C. Lee, and C.-C. Wu, "Scheduling problems with deteriorating jobs and learning effects including proportional setup times," Computers and Industrial Engineering, vol. 58, no. 2, pp. 326-331, 2010.

[25] W.-H. Kuo, "Single-machine group scheduling with timedependent learning effect and position-based setup time learning effect," Annals of Operations Research, vol. 196, no. 1, pp. 349-359, 2012.

[26] S.-J. Yang, "Single-machine scheduling problems simultaneously with deterioration and learning effects under deteriorating multi-maintenance activities consideration," Computers and Industrial Engineering, vol. 62, no. 1, pp. 271-275, 2012.

[27] W.-C. Lee and C.-C. Wu, "Some single-machine and $m$-machine flowshop scheduling problems with learning considerations," Information Sciences, vol. 179, no. 22, pp. 3885-3892, 2009.

[28] J.-B. Wang and J.-J. Wang, "Scheduling jobs with a general learning effect model," Applied Mathematical Modelling, vol. 37, no. 4, pp. 2364-2373, 2013.

[29] R. L. Graham, E. L. Lawler, J. K. Lenstra, and A. H. G. Rinnooy Kan, "Optimization and approximation in deterministic sequencing and scheduling: a survey," Annals of Discrete Mathematics, vol. 5, pp. 287-326, 1979. 


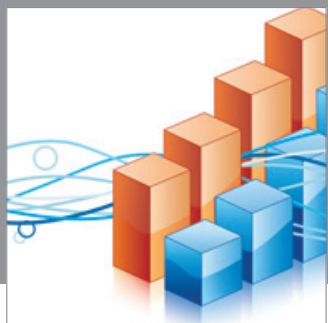

Advances in

Operations Research

mansans

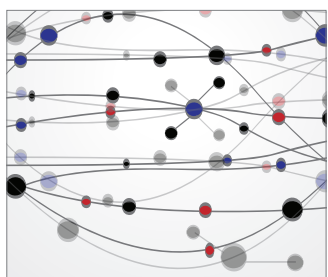

The Scientific World Journal
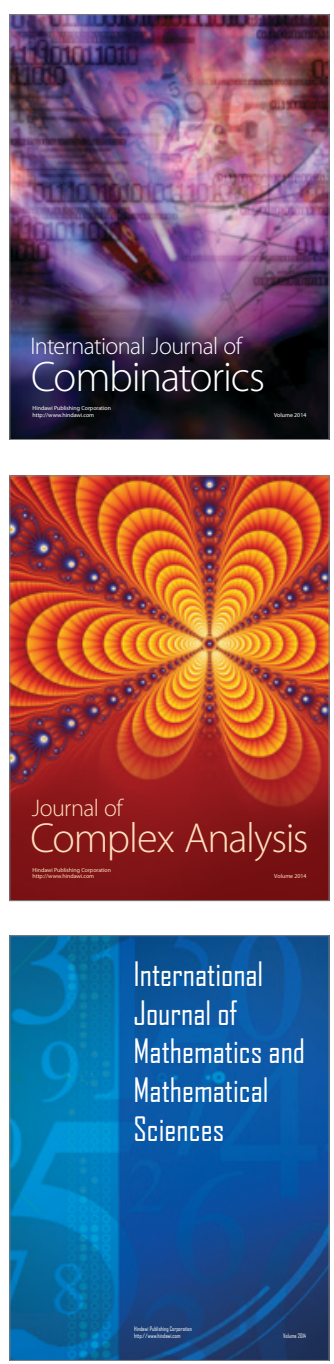
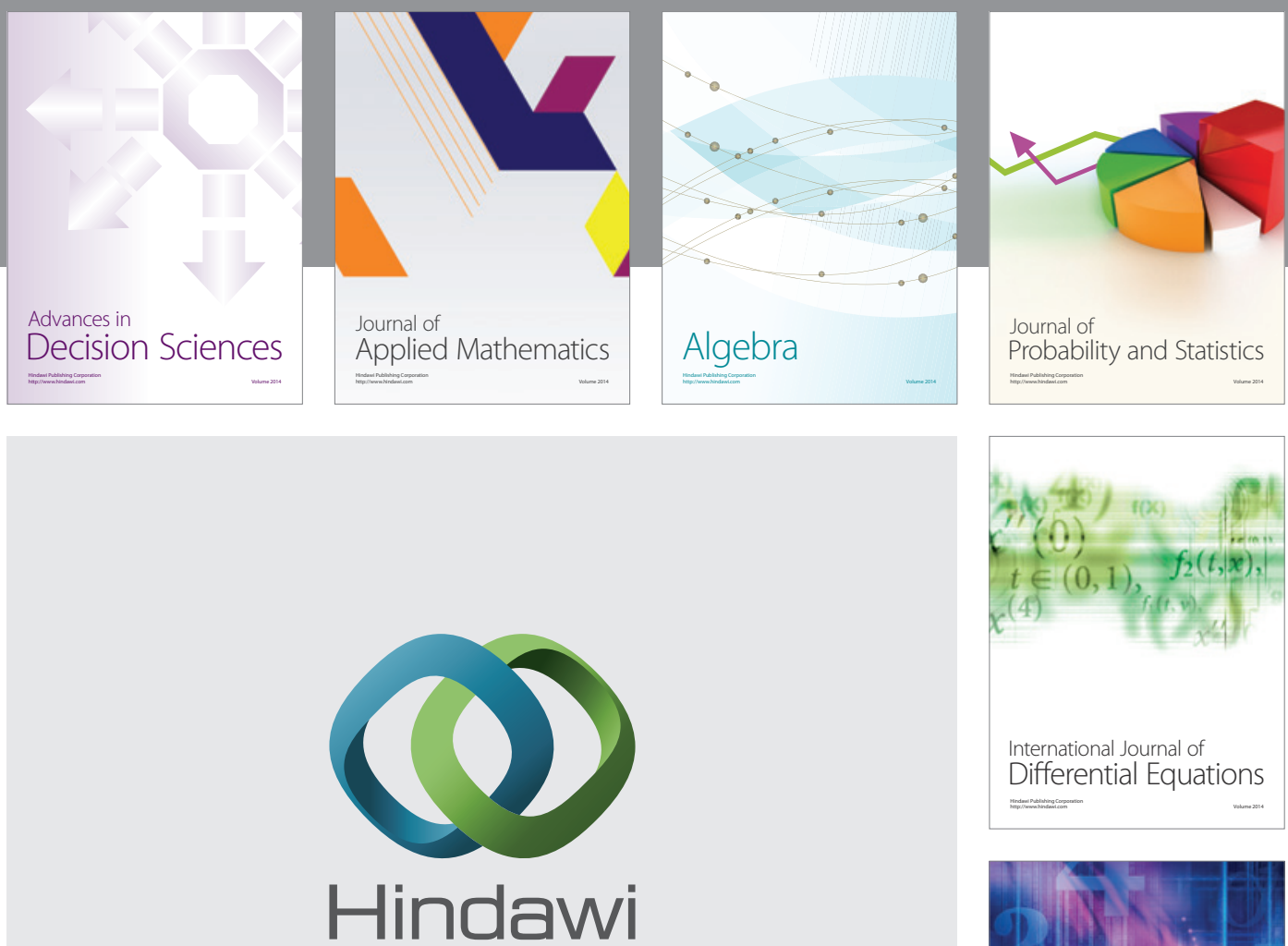

Submit your manuscripts at http://www.hindawi.com
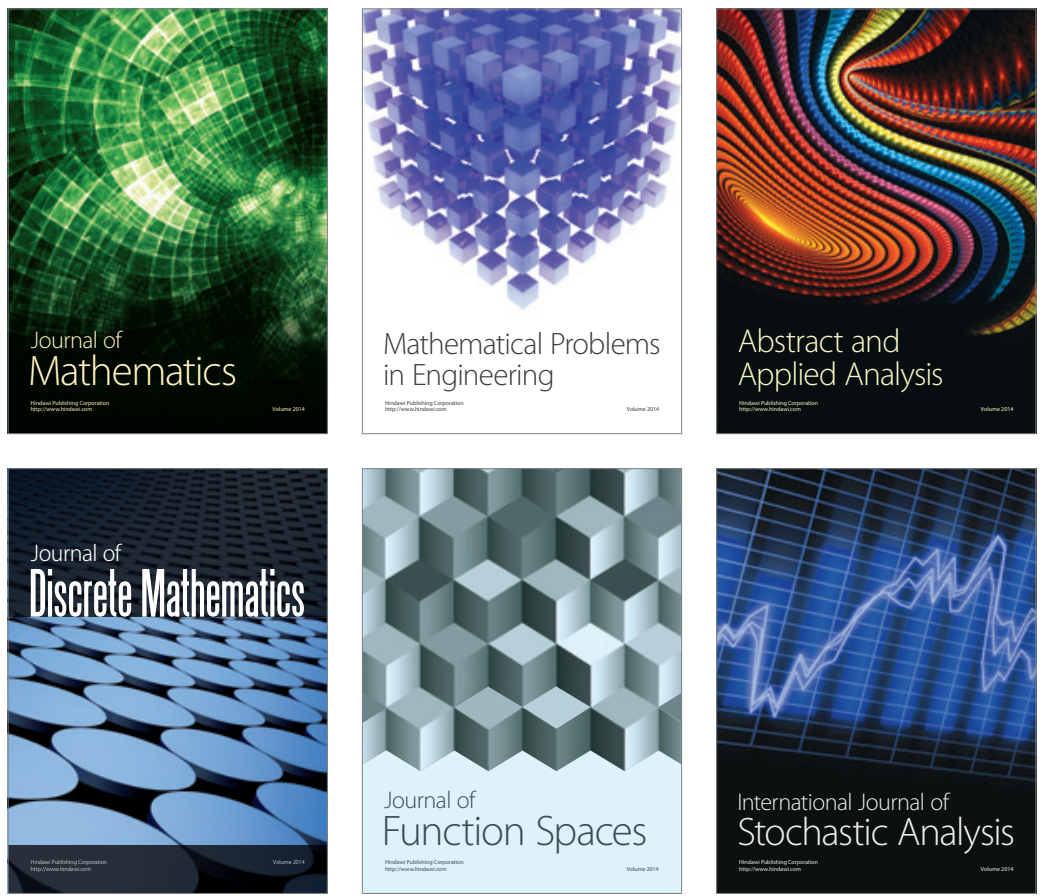

Journal of

Function Spaces

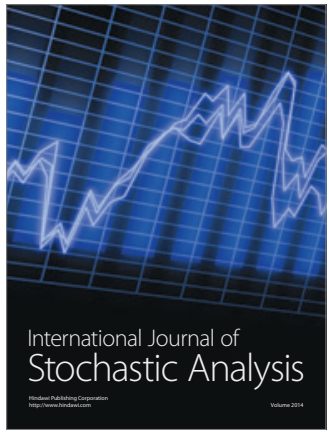

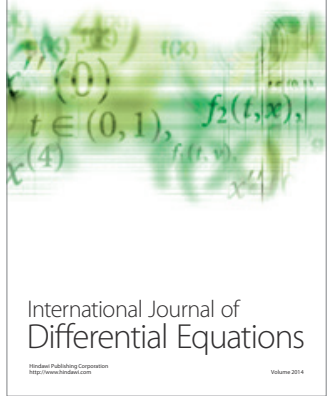
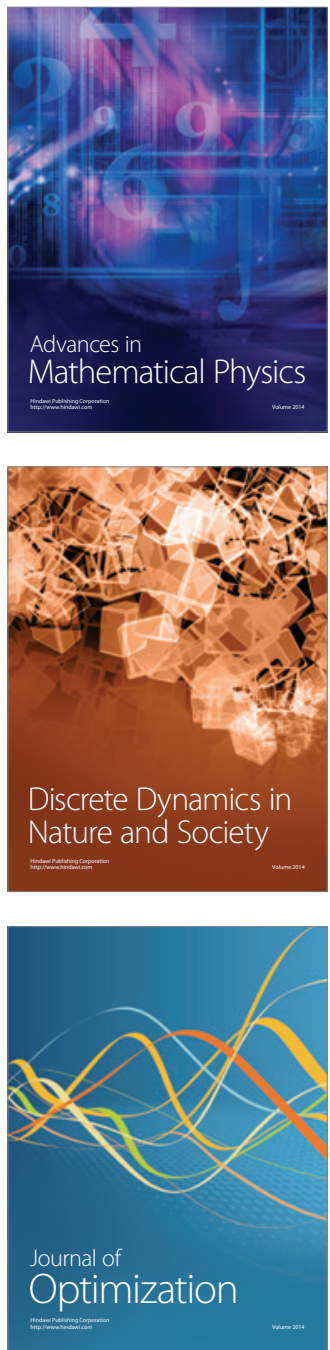